\title{
Management of permanent mandibular second premolar with extraneous canals: An endodontic challenge
}

\author{
Saurabh Gupta1,*, Nabhika Mittal ${ }^{2}$, Saru Dhir ${ }^{3}$, Neha Patil ${ }^{4}$, Manjushree Juneja ${ }^{5}$, Saurabh Juneja ${ }^{6}$ \\ ${ }^{1}$ Professor, Dept. of Conservative Dentistry and Endodontics, D.A.V (C) Dental College and Hospital, Yamunanagar, Haryana, \\ ${ }^{2}$ Private Practitioner, ${ }^{3}$ Senior Lecturer, Dept. of Pedodontics, MM Dental College, Mullana, Haryana, ${ }^{4}$ Senior Lecturer, Dept. of \\ Oral Medicine and Radiology, MGM Dental College, Navi Mumbai, Maharashtra, ${ }^{5,6}$ Reader, ${ }^{5}$ Dept. of Oral Medicine and \\ Radiology, ${ }^{6}$ Dept of Oral Pathology, ITS Centre for Dental Studies \& Research, Murad Nagar, Ghaziabad, Uttar Pradesh, India
}

*Corresponding Author:

Email: drsaurabh.endo@gmail.com

\begin{abstract}
An awareness and understanding of the presence of an additional root and unusual root canal morphology is essential as it determines the successful outcome of endodontic treatment. Aberrations in root canal anatomy are commonly occurring phenomena. The endodontic treatment of mandibular premolar with aberrant root and root canal configuration can be diagnostically and clinically challenging. This article presents a rare anatomic configuration and points to importance of expecting and searching for additional canals because knowledge of their existence would enable clinician to treat a case successfully that otherwise might end in failure.
\end{abstract}

Keywords: Mandibular Second Premolar, Root canal anatomy, Morphological variation.

\section{Introduction}

Pulp canal morphology forms one of the most complex systems in the human body. The internal morphology of tooth varies from person to person both in crown as well as the root. The root canal morphology varies to a great extent in each tooth. Endodontics has reached a level wherein a tooth is considered normal even if variations in their usual anatomy occur. ${ }^{1}$ Successful endodontic therapy is based on three dimensional cleaning, shaping and obturation of complete root canal system. One of the main reasons for endodontic failure is to improper cleaning of root canal system. Root canal systems are formed when secondary dentin deposition occurs during tooth formation. As an anatomic variation, an extra pulp canal is sometimes observed in permanent mandibular premolars where three pulp canals in two roots are seen. ${ }^{2}$ This article presents management of mandibular premolar with anatomic variation having two roots and three pulp canals.

\section{Case Report}

A 35 year old patient reported to the department of Conservative Dentistry and Endodontics with a chief complaint of sensitivity to heat in the right back tooth region. Medical history was noncontributory. On clinical and radiographic examination, a carious lesion in right second premolar encroaching pulp was observed. A diagnosis of irreversible pulpitis was done. A treatment plan of endodontic treatment followed by restoration of the crown was planned. The patient was prepared for endodontic treatment and received local anesthesia of $2 \%$ lidocaine with 1:80,000 epinephrine. A conventional endodontic access opening was done. After removing the coronal pulp and probing with a DG16 endodontic explorer two root canal orifices - buccal and lingual were located. The working length of each canal was estimated by means of an apex locator (Root ZX II: J Morita, Tokyo, Japan). The canals were initially instrumented with \#15 K file (Mani) under irrigation with $5 \%$ sodium hypochlorite. Cleaning and shaping was performed using the crown down technique with nickel-titanium rotary instruments (Universal protaper rotary files, Dentsply, Mallifier). Both the canals were enlarged till file F2. During treatment even after cleaning and shaping of two canals sensitivity was still there. On further examination using radiograph from different angulation (Fig. 1) and dental operating microscope we were able to find a second root with an additional canal. Already prepared two canals were blocked by one size larger gutta percha cone (Fig. 2). The access opening was widened using Gates Gidden drills and cleaning and shaping of third canal was done. Final irrigation with $17 \%$ EDTA followed by $5 \%$ sodium hypochlorite and sealing of root canal space with gutta-percha and ZOE sealer using lateral condensation technique (Fig. 3) and tooth was restored.

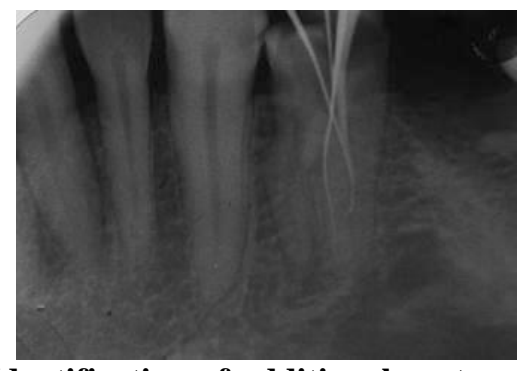

Fig. 1: Identification of additional root canal in the premolar 


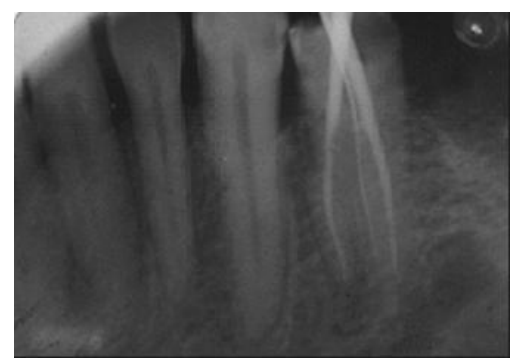

Fig. 2: Obturated buccal and lingual canals

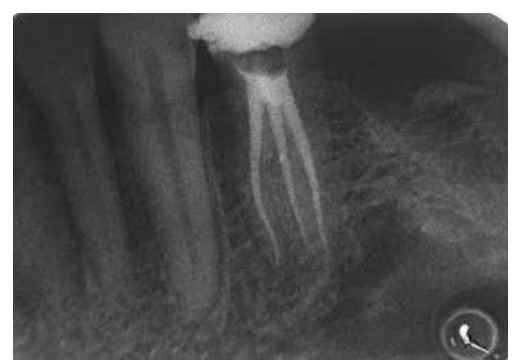

Fig. 3: Obturated additional canals

\section{Discussion}

Success of endodontic treatment is based on complete knowledge of root canal systems and pulpal morphology of teeth. According to Cohen et al., canals are often not treated because they are not located. ${ }^{3}$ Hoen et al. found that in teeth that needed re-treatment the incidence of missed roots or canal was $42 \%{ }^{4}$ Several reports have described the presence of aberrant roots and root canals in mandibular premolar.

The mandibular second premolar is usually a single-rooted tooth with a single root canal system. The root morphology and canal morphology of the mandibular second premolar can be extremely complex and highly variable. The incidence of the number of roots and of the number of canals reported in anatomic studies varies greatly in the literature. The majority of the teeth in various studies had a single root $(99.6 \%)$. Two roots were found in only $0.3 \%$ of the teeth studied. Ingle reported mandibular second premolars have only $12 \%$ chance of a second canal and $0.4 \%$ of a third canal and Wong has reported $11 \%$ possibility of second. ${ }^{5}$ Vertucci $^{6}$ and Zilich ${ }^{7}$ reported the occurrence of three canals in mandibular second premolar at $0.5 \%$ and $0.4 \%$ respectively.

Careful clinical and radiographic examination is prudent for endodontic success in teeth with variation in pulp canal morphology. Adjunctive diagnostic aids such as radiographs from different angulations, clinical probing with endodontic explorers such as DG 16, use of endodontic ultrasonic tips, modification in endodontic access, canal indicator dyes such as $1 \%$ methylene blue, performing the sodium hypochlorite "champagne bubble test," and visualizing canal bleeding points can help in locating extra pulp canals. ${ }^{8}$

Three dimensional radiographic techniques can significantly overcome the limitation of conventional radiography which produces superimposition and two dimensional image and of a three dimensional pulp complex. ${ }^{9}$ Magnification with the use of magnifying loupes and endomicroscopes and fiber-optic transillumination also enhance the ability to search for an extra canal orifice. ${ }^{8}$ Enhanced visualization with better cleaning and shaping of root canal system are definite advantage of endodontic operating microscopes. ${ }^{10}$

\section{Conclusion}

Prerequisite for successful endodontic treatment requires knowledge of root canal anatomy of each tooth as well as numerous atypical ones. Failure of root canal treatment due to inability to find and properly treat root canals can be an avoided with knowledge of existence of these variations in root canal morphology. Thus, it can be concluded that superior endodontic prognosis is directly associated with accurately locating and treating unusual morphology and additional roots and root canals.

\section{References}

1. Bhargavi N, Velmurugan N, Kandaswamy D. The hunt for the elusive canals. Endodontology 2005;17:18-23.

2. Gupta S, Jaiswal S, Arora R. Endodontic management of permanent mandibular left first molar with six root canals. J Contemp Clin Dent 2012;3(suppl 1):S130-3.

3. Cohen S, Burns RC. Pathways of the pulp. 7th ed. Missouri: Elsevier, Mosby; 1998.

4. Hoen MM, Pink FE. Contemporary endodontic retreatments: an analysis based on clinical treatment findings. J Endod 2002;28:834-6.

5. Trope M, Elfenbein L, Tronstad L. Mandibular premolars with more than one root canal in different race groups. $\mathrm{J}$ Endod 1986;12:343-5.

6. Vertucci FJ. Root Canal morphology of mandibular premolars. J Am Dent Assoc 1978;97(1):47-50.

7. Zilich R, Dowson J. Root canal morphology of mandibular first and second premolars. Oral Surg Oral Med Oral Pathol 1973;36:738-44.

8. Ranjan R, Kumar S, Kumar NS, Karunakaran JV. Elusive canals in endodontics. J Ind Acad Dent Specialists 2011;2(2):37-42.

9. Poorni S, Kumar RA, Indira R. Canal complexity of a mandibular first molar. J Conserv Dent 2009;12(1):37-40.

10. Saunders WP, Saunders EM. Conventional endodontics and the operating microscope. Dent Clin North Am 1997;41:415-28. 